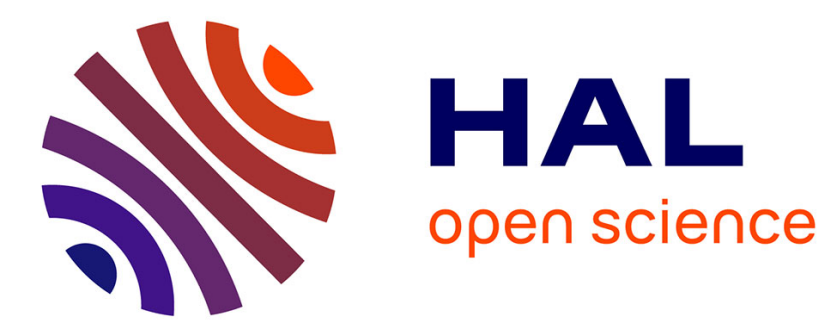

\title{
Demonstration of image-zooming capability for diffractive axicons
}

\author{
Guillaume Druart, Jean Albert Taboury, Nicolas Guérineau, Riad Haïdar, \\ Hervé Sauer, Alain Kattnig, Jérôme Primot
}

\section{- To cite this version:}

Guillaume Druart, Jean Albert Taboury, Nicolas Guérineau, Riad Haïdar, Hervé Sauer, et al.. Demonstration of image-zooming capability for diffractive axicons. Optics Letters, 2008, 33 (4), pp.366-368. 10.1364/OL.33.000366 . hal-00628969

\section{HAL Id: hal-00628969 \\ https://hal-iogs.archives-ouvertes.fr/hal-00628969}

Submitted on 25 May 2012

HAL is a multi-disciplinary open access archive for the deposit and dissemination of scientific research documents, whether they are published or not. The documents may come from teaching and research institutions in France or abroad, or from public or private research centers.
L'archive ouverte pluridisciplinaire $\mathbf{H A L}$, est destinée au dépôt et à la diffusion de documents scientifiques de niveau recherche, publiés ou non, émanant des établissements d'enseignement et de recherche français ou étrangers, des laboratoires publics ou privés. 


\title{
Demonstration of an infrared microcamera inspired by Xenos peckii vision
}

\author{
Guillaume Druart, ${ }^{1, \star}$ Nicolas Guérineau, ${ }^{1}$ Riad Haïdar, ${ }^{1}$ Sophie Thétas, ${ }^{1}$ \\ Jean Taboury, ${ }^{2}$ Sylvain Rommeluère, ${ }^{1}$ Jérôme Primot, ${ }^{1}$ and Manuel Fendler ${ }^{3}$ \\ ${ }^{1}$ ONERA, Chemin de la Hunière, 91761 Palaiseau cedex, France \\ ${ }^{2}$ Institut d'Optique, Campus Polytechnique RD 128, 91127 Palaiseau cedex, France \\ ${ }^{3}$ CEA-LETI/MINATEC, 17 rue des Martyrs, 38054 Grenoble cedex 9, France \\ ${ }^{*}$ Corresponding author: Guillaume.druart@onera.fr
}

Received 12 January 2009; revised 10 March 2009; accepted 13 May 2009; posted 14 May 2009 (Doc. ID 106299); published 10 June 2009

\begin{abstract}
We present an original and compact optical system inspired by the unusual eyes of a Strepsipteran insect called Xenos peckii. It is designed for a field of view of $30^{\circ}$ and is composed of multiple telescopes. An array of prisms of various angles is placed in front of these telescopes in order to set a different field of view for each channel. This type of camera operates in the $[3-5 \mu \mathrm{m}]$ spectral bandwidth and is entirely integrated in a Dewar in order to maximize its compactness. Experimental images are presented to validate this design. (C) 2009 Optical Society of America

OCIS codes: $\quad 110.0110,110.3000,110.3010,110.3080,110.4190,130.3990$.
\end{abstract}

\section{Introduction}

Considerable efforts are now being devoted to inexpensive and miniaturized optical systems for both civilian and military applications because these new developments will open up new market opportunities. For this reason, industrialists are driving their research and development units to design image-capturing systems with reduced size, weight, energy consumption, and cost. However, these constraints are so demanding that the downscaled versions of classical optical systems with a single optical axis are reaching their limits. First, the decrease of the focal length of the lenses tends to increase the difficulties of alignment, which leads to high manufacturing costs. Second, the pixel size of the detectors reaches technological limits and classical optics cannot be downscaled further without losing angular resolution. Nonconventional optical components integrated in nonconventional architectures are thus being proposed to overcome these difficulties.

0003-6935/09/183368-07\$15.00/0

(C) 2009 Optical Society of America
Nonconventional architectures can be found simply by investigating the vision of small invertebrates [1-3]. In order to strike a balance between their small size and the need to have a good vision to navigate, eat, and find a mate, invertebrates have developed compound eyes. Multichannel architecture, inspired by invertebrate vision, offers interesting opportunities in optical design as the principle is to divide the information of the whole scene into several small eyes instead of a single eye. Indeed, in Refs. $[4,5]$, it is demonstrated that combining the information from a flexible array of low-cost cameras can greatly improve performance and even exceed the capability of an individual camera. Moreover, state-of-the-art fabrication and replication technologies for microlens arrays has allowed for the design of compact multichannel architectures in which arrays of lenses can be directly integrated on the detector $[6,7]$. Several teams have proposed various architectures that differ in the way of dividing the information contained in the observation scene. The scene can be spatially split into various channels [6, $\underline{6}-11]$, each channel imaging a small part of the scene. The high-frequency content of the scene can 
be split into different channels also [12,13]. The optical system collects nonredundant undersample images from the same scene. The nonredundancy is usually obtained by subpixel shifts between the images. By applying a superresolution reconstruction on all these small images, a single image with an enhanced angular resolution can be reconstructed [14].

Based on these studies, we have designed a compact multichannel camera called MULTICAM, for the [ $3-5 \mu \mathrm{m}]$ spectral range [15]. The compactness of this system has been obtained by integrating all the optics directly in the Dewar used to cool the detector. The architecture of MULTICAM is inspired by the unusual eyes of a Strepsipteran insect called Xenos peckii. It is made of multiple identical telescopes. An array of prisms of various angles placed in front of the optical system imposes a distinct optical axis for each telescope. Section 2 summarizes the advantages of designing multichannel optical systems, as well as the reason for choosing a microcamera inspired by Xenos peckii vision. In Section 3, the design of MULTICAM will be described. Finally, Section 4 presents the experimental characterization of MULTICAM, and the first image obtained with this camera.

\section{Value of Multichannel Architectures Inspired by Invertebrate Vision}

Traditional optical systems are inspired by singleeye architecture. These systems are based on a single optical axis and a single detector, and the complexity of a diffraction-limited monoaxis camera increases when its field of view and its throughput increase. Besides, in the case of a single-lens system, its angular resolution decreases when the field of view increases.

To address this issue, invertebrates have developed other architectures based on multiaperture optical sensors that enable a wide field-of-view vision with a constant angular resolution. Their eyes are made of multiple simple and well-corrected optical channels, each viewing a small, different part of the global scene. A conceptual comparison of the angular resolution versus the field of view between single and multiple lenses has been made by Sanders and Halford [8] and is recalled in Fig. 1. This graph illustrates the strengths and weaknesses of theses two architecture types, where having multiple apertures is the simplest way to maintain a constant angular resolution over a wide fieldofview and where single-aperture systems are ideal to obtain a high angular resolution.

The compound eyes of the invertebrates can be divided into two main classes [1-3]: apposition compound eyes and superposition compound eyes (Fig. 2). Apposition compound eyes consist of an array of lenses for which each optical channel focuses the light on a small group of photoreceptors [typically fewer than eight photoreceptors; see Fig. 2(a)] Each optical channel is isolated from the others and ob-

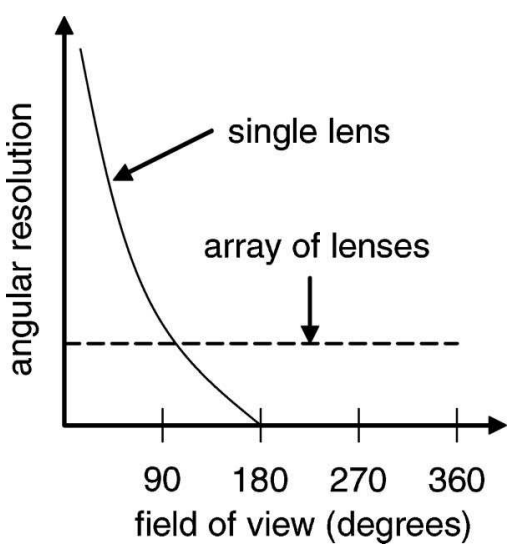

Fig. 1. Comparison of the angular resolution versus the field of view between single and multiple lenses.

serves a unique small solid angle of the scene. This type of compound eye is adapted for diurnal insects. Nocturnal and deep-water invertebrates have developed superposition compound eyes [Fig. 2(b)]. This architecture is made of an array of lenses, too, but unlike the apposition compound eyes, the optical channels are not isolated from each other and a group of lenses can contribute to imaging the same field angle. The array of lenses is thus separated from the retina by a clear zone. This trick allows for enlargement of the pupil of the compound eyes to a dimension greater than the facet of each optical channel. Moreover, superposition eyes produce erect images on the retina. These two types of architecture lead to breakthroughs in optical design $[6,9,10,16,17]$.

These compound eyes can combine a low volume with a large field of view but, unfortunately, this is at the price of a low angular resolution. Indeed, as Lohman demonstrated in Ref. [18], the angular resolution of an optical system decreases if its focal length is reduced when maintaining its stop number, its field of view, and the pixel size of the detector. However, despite their low angular resolution, these high compact architectures can be useful for navigation, detection, and surveillance applications.

Now, if one desires more resolution, another type of compact architecture must be sought. An answer can be found with the amazing visual systems of the males of the Xenos peckii, a Strepsipteran insect, and maybe the Tribolites [2]. The faceted eye of the male Xenos peckii is made of a cluster of large convex lenses $[19,20]$. Each optical unit (an eyelet) has an independent image-forming system with its own retina [Fig. 2(c)]. The field of view of each eyelet has been measured to be around $33^{\circ}$, whereas the intereyelet angle is equal to $27^{\circ}$. Each channel thus has a small amount of overlap with its neighbors. A complete image without spatial lacunarity is then assembled from the neighboring units. This type of eye can be considered to be straddling between simple and compound eyes. The large lenses can admit more light, support more photoreceptors, and thus permit a higher angular resolution with better 


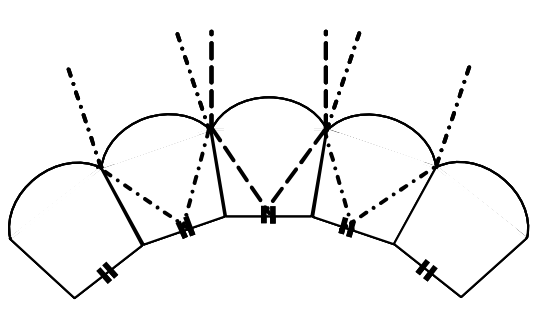

(a)

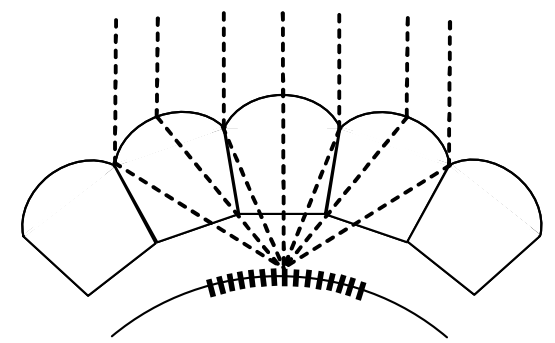

(b)

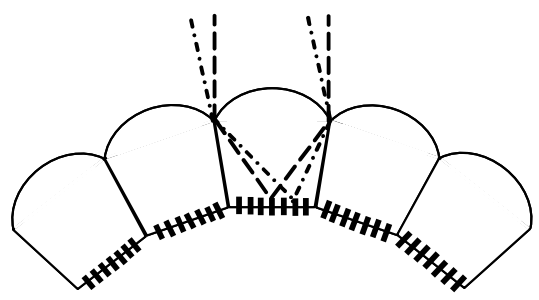

(c)

Fig. 2. Illustration of different types of natural eyes of invertebrates: (a) apposition compound eyes, (b) superposition compound eyes, and (c) eyelets.

sensitivity. Indeed, if we compare the Xenos peckii with an insect having a comparable size, such as a fruit fly, the Xenos peckii has 50 eyelets with around 100 photoreceptors per eyelet, which gives about 5000 resolved points. The fruit fly has only 700 facets, which gives 700 resolved points. This higher resolution has a price: it requires a larger brain allocation to vision needs [20].

We took advantage of this multichannel architecture to design a compact high-resolution infrared camera with a $30^{\circ}$ field of view and using a $\mathrm{HgCdTe}$ technology cooled detector. The detector has a standard format, i.e., $320 \times 240$ pixels with a pitch of $30 \mu \mathrm{m}$. This leads to a camera with a focal length equal to $13.3 \mathrm{~mm}$. Cooled detectors are usually integrated in a Dewar and the optical architecture is designed outside the Dewar. These constraints unfortunately increase the lengths of the optical systems, the smallest Dewar length currently being around $30 \mathrm{~mm}$. In order to miniaturize this camera, we suggest integrating the optics directly into the Dewar. However, due to the cooling limit of the Dewar, the optical system must be as simple as possible without losing optical performance. We thus decide to pack simple well-corrected cameras viewing the scene in different directions, as suggested in Ref. [4], to create the equivalent of a wide-field camera with a high resolution. Moreover, we took advantage of microlens technologies to tightly pack these cameras and to address the integration issue. The necessary overlapping areas are reduced to a minimum, as we will see in Section 3 , in order to obtain a large number of useful pixels.

\section{Design of a Microcamera Inspired by Xenos peckii Vision}

If we want to design a multichannel optical system, we have to carefully divide the whole solid angle of the scene into different sectors. The ideal case is to divide this latter into adjacent sectors in order to avoid overlap areas. Invertebrates mainly tile this solid angle sphere in nonuniform hexagonal units [8]. This type of architecture is possible thanks to their curved retinas. Curved detectors and arrays of lenses are currently under development [21-24], but these technologies are not yet mature enough and we have to mainly use on-the-shelf components (i.e., planar arrays of microlenses and planar detectors).

The more convenient way to divide the scene with a planar detector is to divide it into squarelike areas. However, squares do not efficiently tile a sphere and overlap areas have to be provided in order to image the whole scene without spatial lacunarity. We must then address two other difficulties: first, we have to tilt the optical axis of each channel by using planar components and then we have to suppress the cross talk between adjacent units. Some solutions to avoid cross talk have been suggested: For optical systems with short focal lengths, a separation layer can be inserted between the microlenses and the detector $[10,12]$. In Ref. [10] a pinhole mask placed on the detector is also used to locally select the desired field. For optical systems with long focal lengths, multiple telescopes, each made of a focusing lens followed by a field lens, a pinhole mask, and a relay lens, have been suggested [25]. An intermediate image of the scene is formed at the field lens where the pinhole mask selects the desired field. The selected field is then imaged again by a relay lens. Despite the fact that an architecture with opaque walls can be simpler than an architecture using three stages of lenses, the choice between these two solutions depends on the ability to make thin and long opaque walls.

Two solutions have been put forward to tilt the optical axis of each optical channel differently. The first solution is to use arrays with different pitches. In Refs. $[6,10]$, an array of microlenses has been combined with an array of pinholes. These two arrays have different pitches so that a different field of view is selected at each channel. In Ref. [25] the three arrays of lenses that constitute the telescopes have a different pitch in order to make different tilted optical axes. Each telescope, therefore, faces in a different direction. The drawback of this architecture is that the lenses are decentered and unwanted off-axis aberrations, such as astigmatism, appear. Various ellipsoidal microlenses are needed to correct these aberrations, which leads to an array of lenses that are more complicated to make [26]. Moreover, tilting the optical axis in such a way tends to tighten the tolerances of the lens array fabrication and assembly. Another solution suggested was to tilt the optical axis of each channel by adding a beam deflector in 
the optical system. This beam deflector can be an array of prisms with different angles [10,12], an array of holograms, or a faceted fiber optic faceplate [27]. Indeed, using a beam deflector can lead to compact systems that would most likely have better tolerances without adding off-axis aberration.

In contrast, we have explored the use of an array of prisms at the front of the optical system in order to use planar lens arrays. According to Section 2, we have designed a microcamera inspired by $\bar{X}$ enos peckii vision in order to maximize the angular resolution. It is made of independent image-forming systems sharing a common detector. This camera is an update of a previous optical system, called MULTICAM, described in Ref. [15]. The MULTICAM camera is entirely integrated in a Dewar and is made of multiple identical optical channels observing the same scene with an angular diameter of $8^{\circ}$. As the focal length of this system is long, we have chosen to design telescopes with three arrays of microlenses and an array of pinholes, as seen in Ref. [25]. The array of pinholes is used to avoid the cross talk between each channel. Each telescope is equivalent to a lens with a stop number equal to 8 and a focal length equal to $13.3 \mathrm{~mm}$. This camera works in the $[3-5 \mu \mathrm{m}]$ spectral bandwidth. We recall that the detector used has a standard format of an infrared focal plane array of $320 \times 256$ pixels with a pitch equal to $30 \mu \mathrm{m}$. By integrating an array of prisms with different angles in front of the telescopes, they face in different directions.

The update of MULTICAM with an array of prisms is illustrated in Fig. 3. The field of view of the updated MULTICAM is thus increased. As explained before, an overlap area between each channel has to be foreseen in order to image the whole scene without spatial lacunarity. In the case of MULTICAM, the overlap area is mainly shaped by the pinholes of the mask. The mask is imaged onto the detector by the relay lens. If the projection of the clear area by the relay lens cannot cover the whole detector, blind areas appear in the detection plane. Square pinholes are ideal since the projection of their clear area can cover the whole detector. From a practical standpoint, due to manufacturing constraints, square pinholes usually have rounded corners and blind areas appear on the detector, as illustrated in Fig. 4 . Obviously, the smaller the radius of this rounded square, the smaller the size of the overlap area. The field of view of each channel that must be allocated for the overlap area can be calculated by the following relation:

$$
\theta=2 \arctan \left(\frac{r}{f} \frac{d^{\prime}}{d}\right)
$$

where $r$ is the radius of the corner of the pinhole mask, $f$ is the focal length of the optical system equal to $13.3 \mathrm{~mm}, d$ is the distance between the pinhole mask and the relay lens, and $d^{\prime}$ is the distance between the relay lens and the detector. In the case of MULTICAM, the radius of the corner of the square pinholes is equal to $100 \mu \mathrm{m}$ and the ratio $d^{\prime} / d$ is equal to 2.45; therefore, the field angle of the overlap area is equal to $2^{\circ}$. The useful field of view of each channel is thus equal to $6^{\circ}$ instead of $8^{\circ}$. To increase the field of view of MULTICAM to $30^{\circ}$, an array of $5 \times 5$ prisms with different angles has been made. Each prism has to make the following field conversion:

$$
\begin{aligned}
& {\left[\left(\theta_{1, x}, \theta_{1, y}\right) ;\left(\theta_{2, x}, \theta_{1, y}\right) ;\left(\theta_{1, x}, \theta_{2, y}\right) ;\left(\theta_{2, x}, \theta_{2, y}\right)\right]} \\
& \quad \rightarrow\left[\left(-\frac{\mathrm{FOV}_{u}}{2},-\frac{\mathrm{FOV}_{u}}{2}\right) ;\left(\frac{\mathrm{FOV}_{u}}{2},-\frac{\mathrm{FOV}_{u}}{2}\right) ;\right. \\
& \left.\quad \times\left(-\frac{\mathrm{FOV}_{u}}{2}, \frac{\mathrm{FOV}_{u}}{2}\right) ;\left(\frac{\mathrm{FOV}_{u}}{2}, \frac{\mathrm{FOV}_{u}}{2}\right)\right]
\end{aligned}
$$

where $\theta_{x}$ is the field angle along the $x$ axis, $\theta_{y}$ is the field angle along the $y$ axis, and $\mathrm{FOV}_{u}$ is the useful field of view of a channel. $\mathrm{FOV}_{u}$ is given by the

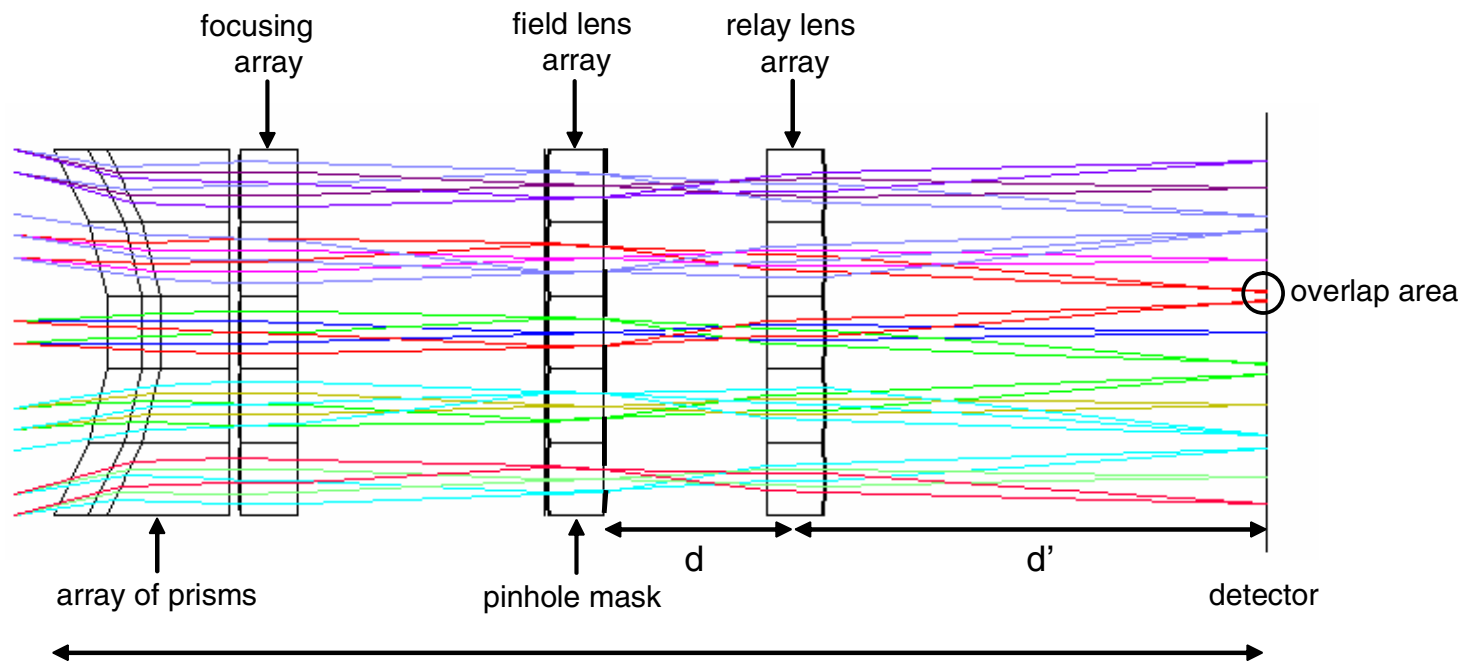

$24.8 \mathrm{~mm}$

Fig. 3. (Color online) Illustration of the update of MULTICAM with an array of prisms. 


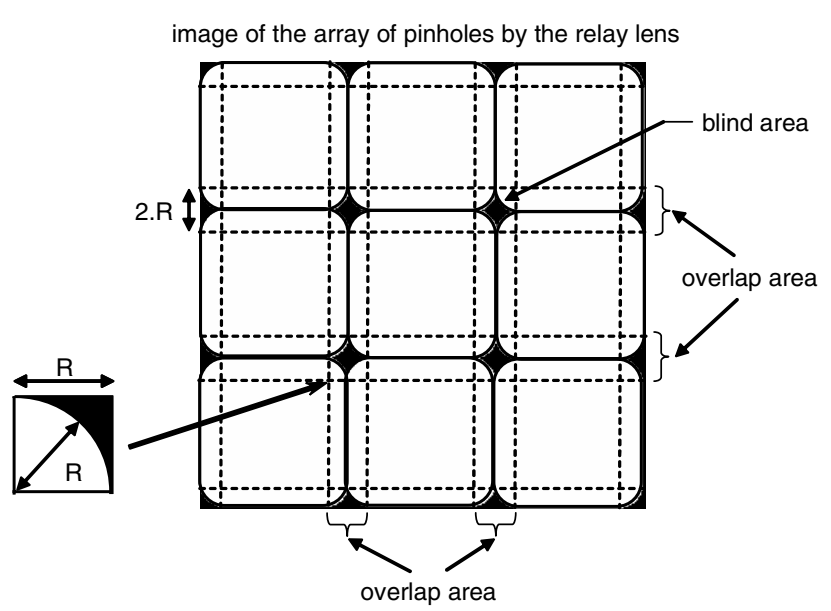

Fig. 4. Projection by the relay lens of the clear area of the pinhole mask on the detector. The radius $R$ is equal to $r . d^{\prime} / d$, where $r$ is the radius of the corner of the pinhole mask, $d$ is the distance between the pinhole mask and the relay lens, and $d^{\prime}$ is the distance between the relay lens and the detector.

relation $\mathrm{FOV}_{t}-\mathrm{FOV}_{o}$, where $\mathrm{FOV}_{t}$ is the total field of view of each channel and $\mathrm{FOV}_{o}$ is the field of view of the overlap area. In our case, $\mathrm{FOV}_{t}=8^{\circ}$, $\mathrm{FOV}_{o}=2^{\circ}$, and, thus, $\mathrm{FOV}_{u}=6^{\circ}$. Moreover, angles $\theta_{1}$ and $\theta_{2}$ must confirm the relation $\left|\theta_{2}-\theta_{1}\right|=$ $\mathrm{FOV}_{u}$. The angles $A_{x / y}$ along the $x$ axis and $y$ axis of each prism have to be chosen so that the angular deviation of the prism in each direction is equal to $\left(\theta_{1, x / y}+\theta_{2, x / y}\right) / 2$. Therefore, the angles $A_{x / y}$ must confirm the relation as follows:

$$
\sin \left(\left|\frac{\theta_{1, x / y}+\theta_{2, x / y}}{2}\right|+A_{x / y}\right)=\sin \left(A_{x / y}\right)
$$

The various prisms were made separately and then assembled. They are made of $\mathrm{CaF}_{2}$. The array of prisms has been added in front of MULTICAM, as illustrated in Fig. 5, and the whole optical system has been integrated on the cold shield in the Dewar, as illustrated in Fig. $\underline{6}$.

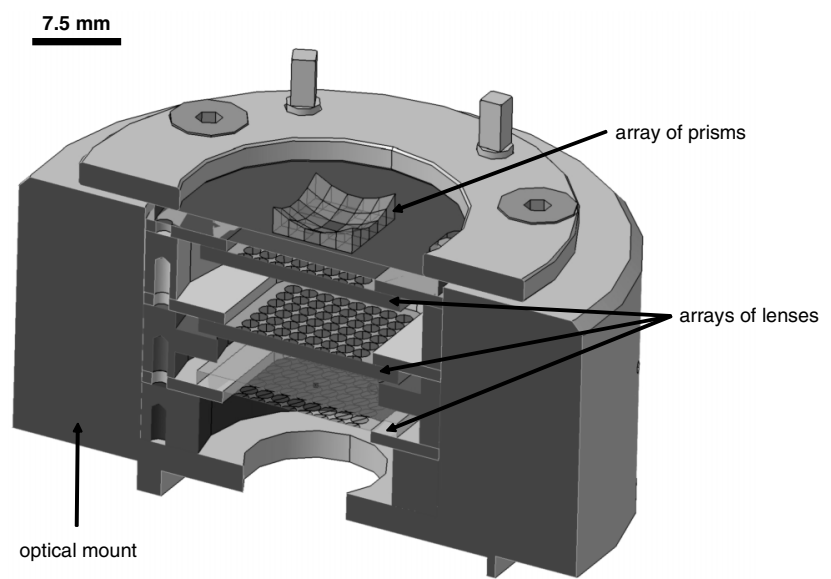

Fig. 5. Illustration of the implementation of the optics in a mechanical mount.

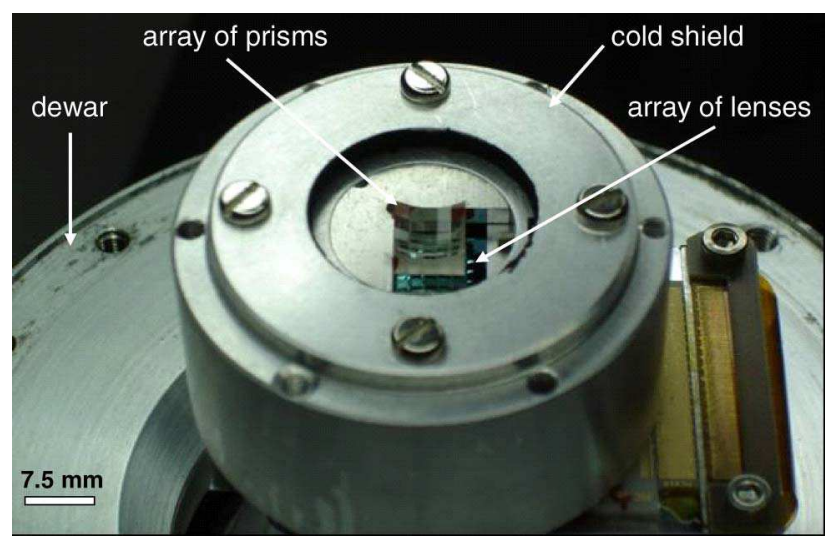

Fig. 6. (Color online) Integration of MULTICAM with an array of prisms in a Dewar.

\section{Characterization of MULTICAM Updated With an Array of Prisms}

The point spread function (PSF) has been measured for each channel in order to evaluate the image quality of the whole field, using the test bench illustrated in Fig. 7. A point source, which is not resolved by the optical system, has been imaged at the center of each optical channel. A pinhole placed in the focal plane of a collimator and illuminated by a blackbody at $1200^{\circ} \mathrm{C}$ makes the point source. The field angle of the point source can be changed by rotating either the collimator/ blackbody unit $\left(\theta_{y}\right)$ or the mirror $\left(\theta_{x}\right)$.

The experimental PSFs for each channel of MULTICAM with an array of prisms are illustrated in Fig. 8 . The point source used for this experiment has an angular diameter of $0.03^{\circ}$, which is less than the angular resolution of the optical system (expected to be $0.12^{\circ}$ ). These measurements have thus been made at the diffraction limit of this system. No filter has been used, so that the camera is working with a wide spectral bandwidth. We notice that the PSF width increases from the center to the side of the array. The optical quality of the channels at the array side is indeed more degraded by the lateral chromatic aberrations caused by a more significant deviation of the prisms.

In order to check that the deterioration of the PSF is due to chromatic aberrations, we acquired two PSFs at the center of channel 1 (see Fig. 8) with and without a bandpass filter. The spectral

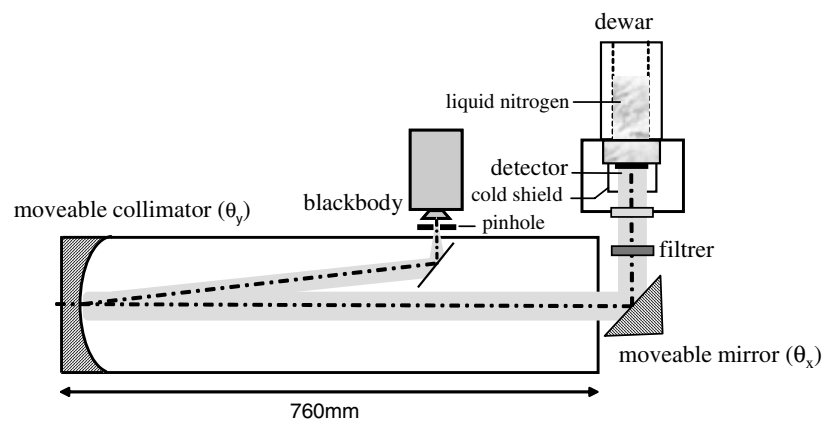

Fig. 7. Illustration of the experimental setup for the characterization of MULTICAM with an array of prisms. 


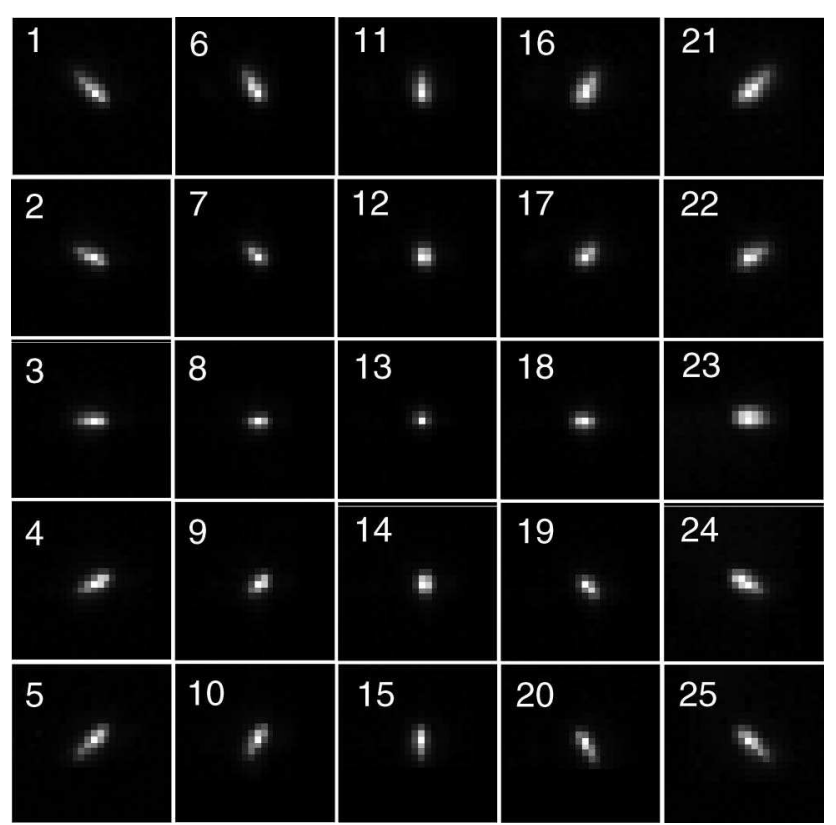

Fig. 8. Measurement of the PSF for each channel of MULTICAM with an array of prisms.

bandwidth of the filter is equal to [4.08-4.728 $\mu \mathrm{m}]$. These two PSFs are illustrated in Fig. 9, which actually shows that the optical quality of the channel is increased by restricting the spectral bandwidth of the camera. Several methods can be used to reduce the chromatic aberrations induced by the prisms. Prisms with a higher index of refraction are usually less dispersive (for example, Ge or $\mathrm{Si}$ ). However, they require an antireflection coating, and this makes the manufacturing process more difficult. The array of prisms can be modified by an array of grisms, which
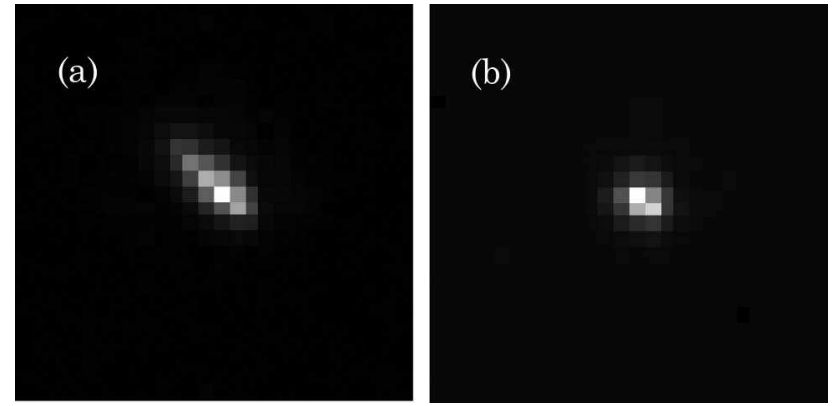

Fig. 9. Comparison of two PSFs acquired at the center of channel 1 (see Fig. 8) (b) with or (a) without a bandpass filter.

are a combination of a prism and a grating to minimize chromatic aberration [28].

An image produced by the update of MULTICAM with an array of prisms is given in Fig. 10(a). We confirmed that all the telescopes observe a different field of view and that the whole field of view of the camera is thus increased thanks to the array of prisms. As we have seen in Section 3, overlap areas have been provided for in order to compensate for the presence of the blind spots, which can easily be observed in Fig. 10(a). The overlap areas can also be observed. Anomalous replication of glasses and of fingers can be seen. Image processing is thus necessary in order to recover the whole scene. We have estimated the shifts between the small images experimentally. To do this, we acquired different point sources in the different overlap areas where four adjacent telescopes can see the same source point. These overlap areas are located around the blind areas (see Fig. 4). Figure 10(b) shows the different point sources we used to estimate the shifts between each channel. The useful field of view of each channel was then (a)

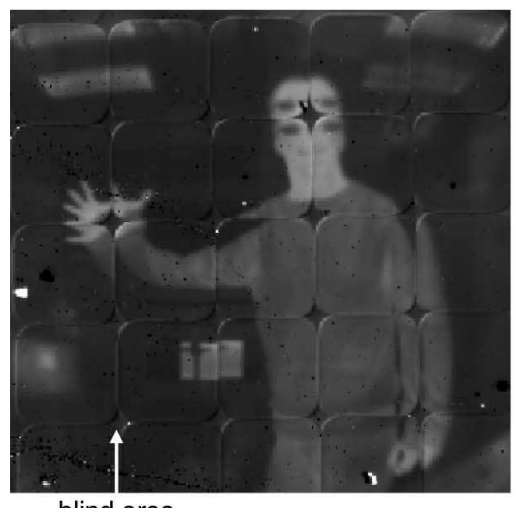

blind area (b)
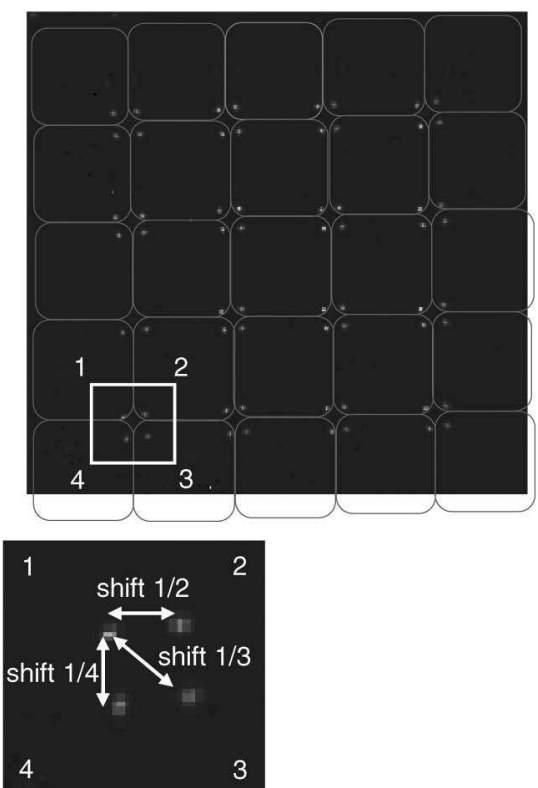

(c)

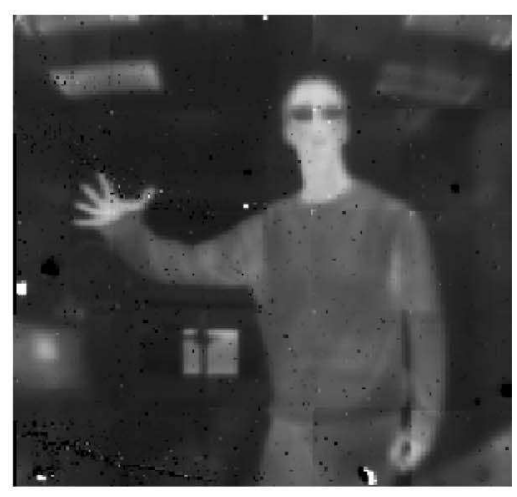

Fig. 10. (a) Illustration of an image acquired by MULTICAM with an array of prisms, (b) assessment of the shifts between each channel, and (c) restoration of the whole scene. 
extracted and, thanks to the shift values obtained previously, the whole scene observed by the camera was recovered [see Fig. 10(c)]. As the overlap area was well established, there are no spatial lacunarities in the observation scene.

\section{Conclusion}

Multichannel optical systems modeled on invertebrate vision are simple and compact systems with constant angular resolution over a large field of view. We believe that the eyes of the Xenos peckii offer the best multichannel configuration for maximizing angular resolution. A compact infrared camera based on the vision of the Xenos peckii and entirely integrated in a Dewar has been designed. It is made of multiple telescopes and an array of prisms of different angles. Thanks to these prisms, each telescope sees a different field of view. The overall field of view of this compact camera is equal to $30^{\circ}$. Overlap areas have, however, been taken into account in order to restore the whole scene without spatial lacunarity.

This camera has been made and characterized experimentally. Because of fabrication constraints, the chromatic aberrations were not corrected and they appear at the side of the camera because of the high dispersive power of the prisms and because of their high angles on the sides. Some solutions have been proposed to correct these aberrations.

Last, an image of this camera was acquired and, after calibration, image processing was proposed to reconstruct the whole scene. Thanks to the overlap areas, no spatial lacunarity is observed on the image.

This work was sponsored by the Délégation Générale de l'Armement (DGA) of the French Ministry of Defense.

\section{References}

1. R. Völkel, "Natural optical design for microcameras," Laser Optoelektron. 30, 47-55 (1998).

2. M. F. Land and D.-E. Nilsson, Animal Eyes, Oxford Animal Biology Series (Oxford U. Press, 2002).

3. E. Warrant and D.-E. Nilsson, Invertebrate Vision (Cambridge U. Press 2006).

4. B. Wilburn, N. Joshi, V. Vaish, E.-V. Talvala, E. Antunez, A. Barth, A. Adams, M. Horowitz, and M. Levoy, "High performance imaging using large camera arrays," ACM Trans. Graph. 24, 765-776 (2005).

5. P. M. Shankar, W. C. Hasenplaugh, R. L. Morrison, R. A. Stack, and M. A. Neifeld, "Multiaperture imaging," Appl. Opt. 45, 2871-2883 (2006).

6. J. Duparré, P. Dannberg, P. Shreiber, A. Bräuer, and A. Tünnermann, "Artificial apposition compound eye fabricated by micro-optics technology," Appl. Opt. 43, 4303-4310 (2004).

7. K. Fife, A. El Gamal, and H.-S. P. Wong, "A 3D multi-aperture image sensor architecture," in Proceedings of Custom Integrated Circuits Conference, 2006 (IEEE, 2006), pp. 281-284.

8. J. S. Sanders and C. E. Halford, "Design and analysis of apposition compound eye optical sensors," Opt. Eng. 34, 222-235 (1995).
9. K. Hamanaka and H. Koshi, "An artificial compound eye using a microlens array and its application to scale-invariant processing," Opt. Rev. 3, 264-268 (1996).

10. J. Duparré, P. Dannberg, P. Schreiber, A. Bräuer, and A. Tünnermann, "Thin compound-eye camera," Appl. Opt. 44, 2949-2956 (2005).

11. J. W. Duparré and F. C. Wippermann, "Micro-optical artificial compound eyes," Bioinspir. Biomim. 1, R1-R16 (2006).

12. J. Tanida, T. Kumagai, K. Yamada, S. Miyatake, K. Ishida, T. Morimoto, N. Kondou, D. Miyazaki, and Y. Ichioka, "Thin observation module by bound optics (TOMBO): concept and experimental verification,” Appl. Opt. 40, 1806-1813 (2001).

13. M. Shankar, R. Willet, N. Pitsianis, T. Schulz, R. Gibbons, R. T. Kolste, J. Carriere, C. Chen, D. Prather, and D. Brady, "Thin infrared imaging systems through multichannel sampling," Appl. Opt. 47, B1-B10 (2008).

14. Y.Kitamura,R.Shogenji,K.Yamada,S.Miyatake,M.Miyamoto, T. Morimoto, Y. Masaki, N. Kondou, D. Miyazaki, J. Tanida, and Y. Ichioka, "Reconstruction of a high-resolution image on a compound-eye image-capturing system," Appl. Opt. 43, 1719-1727 (2004).

15. G. Druart, N. Guérineau, R. Haïdar, E. Lambert, M. Tauvy, S. Thétas, S. Rommeluère, J. Primot, and J. Deschamps, "MULTICAM: a miniature cryogenic camera for infrared detection," Proc. SPIE 6992, 699215 (2008).

16. C. Hembd-Sölner, R. F. Stevens, and M. C. Hutley, "Imaging properties of the Gabor superlens," J. Opt. A Pure Appl. Opt. 1, 94-102 (1999).

17. V. Gubsky, M. Gertsenshteyn, and T. Jannson, "Lobster-eye infrared focusing optics," Proc. SPIE 6295 62950F (2006).

18. A. W. Lohmann, "Scaling laws for lens systems," Appl. Opt. 28, 4996-4998 (1989).

19. E. K. Buschbeck, B. Ehmer, and R. R. Hoy, "Chunk versus point sampling: visual imaging in a small insect," Science 286, 1178-1180 (1999).

20. E. K. Buschbeck, B. Ehmer, and R. R. Hoy, "The unusual visual system of the Strepsiptera: external eye and neuropils," J. Comp. Physiol. A 189, 617-630 (2003).

21. S.-B. Rim, P. B. Catrysse, R. Dinyari, K. Huang, and P. Peumans, "The optical advantages of curved focal plane arrays," Opt. Express 16, 4965-4971 (2008).

22. H. C. Ko, M. P. Stoykovich, J. Song, V. Malyarchuk, W. M. Choi, C.-J. Yu, J. B. Geddes III, J. Xiao, S. Wang, Y. Huang, and J. A. Rogers, "A hemispherical electronic eye camera based on compressible silicon optoelectronics," Nature 454, 748-752 (2008).

23. K.-H. Jeong, J. Kim, and L. P. Lee, "Biologically inspired artificial compound eyes," Science 312, 557-561 (2006).

24. R. J. Martín-Palma, C. G. Pantano, and A. Lakhtakia, "Replication of fly eyes by the conformal-evaporated-filmby-rotation technique," Science 312, 557-561 (2006).

25. J. Duparré, P. Schreiber, A. Matthes, E. Pshenay-Severin, A. Bräuer, A. Tünnermann, R. Völkel, M. Eisner, and T. Sharf, "Microoptical telescope compound eye," Opt. Express 13, 889-901 (2005).

26. J. Duparré, F. Wippermann, P. Dannberg, and A. Reimann, "Chirped arrays of refractive ellipsoidal microlenses for aberration correction under oblique incidence," Opt. Express 13, 10539-10550 (2005).

27. L. C. Laycock and V. A. Handerek, "Multi-aperture imaging device for airborne platforms," Proc. SPIE 6737, 673709 (2007).

28. C. B. Chen, "Beam steering and pointing with counter-rotating grisms," Proc. SPIE 6714, 671409 (2007). 